\title{
A Compensating Anastigmatic Submillimeter Array Imaging System for STEAMR
}

\author{
Mark Whale, Member, IEEE, Anthony Murphy, Member, IEEE, Axel Murk, Matthias Renker, Urban Frisk, and
} Olle Janson

\begin{abstract}
In this paper, we present a novel technique for the removal of astigmatism in submillimeter-wave optical systems through employment of a specific combination of so-called astigmatic off-axis reflectors. This technique treats an orthogonally astigmatic beam using skew Gaussian beam analysis, from which an anastigmatic imaging network is derived. The resultant beam is considered truly stigmatic, with all Gaussian beam parameters in the orthogonal directions being matched. This is thus considered an improvement over previous techniques wherein a beam corrected for astigmatism has only the orthogonal beam amplitude radii matched, with phase shift and phase radius of curvature not considered. This technique is computationally efficient, negating the requirement for computationally intensive numerical analysis of shaped reflector surfaces. The required optical surfaces are also relatively simple to implement compared to such numerically optimized shaped surfaces. This technique is implemented in this work as part of the complete optics train for the STEAMR antenna. The STEAMR instrument is envisaged as a mutli-beam limb sounding instrument operating at submillimeter wavelengths. The antenna optics arrangement for this instrument uses multiple off-axis reflectors to control the incident radiation and couple them to their corresponding receiver feeds. An anastigmatic imaging network is successfully implemented into an optical model of this antenna, and the resultant design ensures optimal imaging of the beams to the corresponding feed horns. This example also addresses the challenges of imaging in multi-beam antenna systems.
\end{abstract}

Index Terms-ABCD matrices, anastigmatic, astigmatic, Gaussian beams, multi-beam, quasi-optical, STEAMR.

\section{INTRODUCTION}

$\mathbf{I}$ $\mathrm{N}$ THIS PAPER, we address the primary optical design issue of removing the astigmatism typically inherent in submillimeter-wave limb sounders. This is an important aspect of submillimeter-wave limb sounding instruments which utilize asymmetric beam amplitude patterns, typically elliptical, to observe the atmosphere [1], [2]. In terms of imaging within the receiving antenna optics, this elliptical beam pattern is considered

Manuscript received June 19, 2012; revised September 06, 2012; accepted November 06, 2012. Date of publication December 28, 2012; date of current version February 07, 2013. This work was supported by the Swiss National Science Foundation under Grant 200021-130517 and by the ESA PRODEX under Project C4000100544.

M. Whale, A. Murk and M. Renker are with the Institute for Applied Physics, University of Bern, Bern 3012, Switzerland (e-mail: whale@iap.unibe.ch).

A. Murphy is with the Experimental Physics Department, National University of Ireland, Maynooth, Co. Kildare, Ireland.

U. Frisk is with Omnisys Instruments AB, Västra Frölunda, S-42130, Sweden.

O. Janson is with the Swedish Space Corporation, Solna, SE-171 04, Sweden.

Color versions of one or more of the figures in this paper are available online at http://ieeexplore.ieee.org.

Digital Object Identifier 10.1109/TTHZ.2012.2228895 orthogonally astigmatic in terms of a skew Gaussian beam treatment [3]. Previous techniques for the correction of this astigmatism in either antenna relay optics [2] or in laboratory imaging arrangements [4] have considered matching of the orthogonal Gaussian beam amplitude radii, with the phase radius of curvature and phase shift not being considered. The beam can then be said to not be truly stigmatic, as the complex beam parameters for the orthogonal directions are not equivalent. We present here a technique for the optical correction of orthogonally astigmatic Gaussian beams, with the resultant beam being consolidated in the orthogonal directions.

The theoretical application of Gaussian beam theory to generate an anastigmatic imaging network is presented in Section II. In Section III an overview of the antenna optics scheme for the STEAMR instrument is given, which includes a summary of the instrument's observation goals and the consequent optical requirements. Further detailed in this section is the specific solution of the anastigmatic imaging network as it applies to the STEAMR antenna optics. An overview of the off-axis astigmatic reflectors required for physical implementation of this imaging network is presented in Section IV. The focal plane array developed for the imaging of the individual beams to their corresponding receiver elements is described in Section V. In Section VI the results of electromagnetic simulations of the STEAMR optical arrangement performed through the reflector design and simulation software package GRASP are presented. Finally, based upon the results of the analytical and numerical simulations presented here we draw conclusions with regard the success and applicability of this optical design to the STEAMR antenna.

\section{Correction of an Astigmatic Gaussian Beam}

The analytical technique of modeling quasioptical systems using Gaussian beam analysis through $A B C D$ matrices is well founded [5]. This technique provides an efficient and accurate first order approximation for modeling the propagation of electromagnetic fields through submillimeter-wave optical systems composed of reflectors and/or lenses, providing that aberration and truncation effects are negligible and that the receiver antennas maintain aperture distributions which can be efficiently described in terms of Gaussian beams, such as corrugated feed horns [6]. The normalized electric field distribution of the fundamental Gaussian beam mode in cylindrical coordinates is given as

$$
\begin{aligned}
& E(r, z)= \\
& \sqrt{\frac{2}{\pi w(z)^{2}}} \times \exp \left(\frac{-r^{2}}{w(z)^{2}}-j k z-\frac{j \pi r^{2}}{\lambda R(z)}+j \phi_{0}(z)\right)
\end{aligned}
$$


where $w(z)$ is the beam radius parameter at a distance $z$ from the beam waist location where $w(0)=w_{0}, R(z)$ is the phase radius of curvature parameter at a distance $z$ from the beam waist location, where $R(0)=\infty$ and $\phi_{0}(z)$ is the longitudinal phase shift, or Guoy phase shift, and $r$ is the radial coordinate measured from the central propagation axis. The beam parameters of $w(z), R(z)$, and $\phi(z)$ are given by the following equations:

$$
\begin{aligned}
w(z) & =w_{0} \sqrt{1+\left(\frac{\lambda z}{\pi w_{0}^{2}}\right)^{2}} \\
R(z) & =z+\frac{1}{z}\left(\frac{\pi w_{0}^{2}}{\lambda}\right)^{2} \\
\phi_{0}(z) & =\arctan \left(\frac{\lambda z}{\pi w_{0}^{2}}\right) .
\end{aligned}
$$

While these equations will describe the evolution of the paraxial fundamental Gaussian beam parameters in unhindered free-space propagation, ray transfer or $A B C D$ matrices are required to describe the beam parameter evolution through a system composed of optical elements such as reflectors, lenses or dielectric interfaces. For a general optical system composed of $N$ components, the individual $A B C D$ matrices that describe these components are cascaded together to form the complete system $A B C D$ matrix. The $A B C D$ matrices of the individual components have simple forms [5].

A Gaussian beam can be treated as a complex point source with a phase radius of curvature that evolves in the same way as the wavefront radius of a geometrical point source. Defining the complex beam parameter $q(z)$ as

$$
\frac{1}{q(z)}=\frac{1}{R(z)}-\frac{j \lambda}{\pi w(z)^{2}}
$$

allows for the following calculation of the output complex beam parameter:

$$
q_{\mathrm{out}}=\frac{A q_{\mathrm{in}}+B}{C q_{\mathrm{in}}+D}
$$

from which the beam parameters $w$ and $R$ of the beam at the output plane can be readily calculated from (5).

In the treatment of astigmatic Gaussian beams, we simplify the general approach taken in [7], by reducing the definition of a beam to orthogonal or simple astigmatism, wherein a skew ray treatment requires that the Gaussian beam parameters for the two transverse orthogonal directions are mismatched, i.e., $w_{\mathrm{Az}} \neq w_{\mathrm{El}}, R_{\mathrm{Az}} \neq R_{\mathrm{El}}$, and $\phi_{\mathrm{Az}} \neq \phi_{\mathrm{El}}$, where the subscripts $A z$ and $E l$ refer to azimuth and elevation. In this treatment the beam is thus defined with two sets of independently evolving Gaussian beam parameters. In this work, we expand upon previous works addressing this issue [4], [8]-[10], where astigmatism is defined solely in terms of mismatched beam amplitude radii in the transverse orthogonal directions, with the mismatched phase radii of curvature not being addressed. The resultant beams can thus not be considered as truly stigmatic i.e., where $w_{\mathrm{Az}}=w_{\mathrm{El}}, \phi_{\mathrm{Az}}=\phi_{\mathrm{El}}$, and $R_{\mathrm{Az}}=R_{\mathrm{El}}$. In order to produce the desired stigmatic beam, more degrees of freedom are required in the optical train than those put forth in [4], [8]-[10], all of which utilize only a single phase transforming element.

The incident beam is treated as a simple astigmatic Gaussian, with independently evolving beam parameters
$\left(w_{\mathrm{Az}}, \phi_{\mathrm{Az}}, R_{\mathrm{Az}}\right),\left(w_{\mathrm{El}}, \phi_{\mathrm{El}}, R_{\mathrm{El}}\right)$. An anastigmatic imaging network can then be developed using ABCD matrix analysis in order to generate an output beam with the required matched beam parameters. For a system composed of multiple phase transforming elements, either lenses or reflectors, there are consequently multiple degrees of freedom such as the focal lengths of the components $f_{i}$ and/or the separation of the components $z_{i j}$ available to manipulate the evolution of the propagating beam. These degrees of freedom are represented by the independent $A B C D$ matrix coefficient values of the general optical system. The combination of reflectors and focal lengths that corrects this simple astigmatism is referred to here on as the anastigmatic imaging network.

The propagation distances are common to both orthogonal directions, while the focal lengths are independent: $\left(f_{n-A z}, f_{n-E l}\right)$. This thus establishes a requirement for a minimum of three reflector elements for the anastigmatic imaging network in order to have sufficient degrees of freedom. First consider the $A B C D$ matrix $M$ that describes the optical system between an input plane co-located with the first reflector and an output plane co-located with the third and last reflector:

$$
M=\left(\begin{array}{ll}
A & B \\
C & D
\end{array}\right)
$$

where

$$
\begin{aligned}
& A=1-\frac{z_{1}+z_{2}}{f_{1}}-\frac{z_{2}}{f_{2}}+\frac{z_{1} z_{2}}{f_{1} f_{2}} \\
& B=z_{1}+z_{2}-\frac{z_{1} z_{2}}{f_{2}} \\
& C=-\frac{1}{f_{1}}-\frac{1}{f_{2}}-\frac{1}{f_{3}}+\frac{z_{1}+z_{2}}{f_{3}}\left(\frac{1}{f_{1}}+\frac{1}{f_{2}}\right)+\frac{z_{1} z_{2}}{f_{1} f_{2} f_{3}} \\
& D=1-\frac{z_{1}}{f_{2}}-\frac{z_{1}+z_{2}}{f_{3}}+\frac{z_{1} z_{2}}{f_{2} f_{3}}
\end{aligned}
$$

where $f_{i}$ are the focal lengths of the component reflectors, $z_{1}$ is the propagation distance between the first and second reflectors and $z_{2}$ is the propagation distance between the second and third reflectors. Setting the $B$ component of the above $A B C D$ matrix to zero corresponds the formation at the output plane of an image of the input plane. From this stipulation the following can be inferred from (9):

$$
f_{2}=\frac{z_{1} z_{2}}{z_{1}+z_{2}}
$$

from which it is then trivial to determine $f_{1}$ and $f_{3}$. This result can then be readily applied to determine the focal lengths of the component reflectors in the two orthogonal directions. For the two axis directions three degrees of freedom are required, so that the minimum number of optical components required to achieve the anastigmatic network, provided that the inter-reflector propagation distances are fixed, is three. In the resultant system this will require that each reflector have the correspondingly differing focal lengths $\left(f_{n-A z}, f_{n-E l}\right)$ for the principle orthogonal directions of the skew beams. The design of such biconic reflectors will be described in Section IV.

\section{STEAMR ANTENNA OPTICS}

The STEAMR instrument is a Swedish national contribution to the upcoming ESA PREMIER mission, which is a candi- 


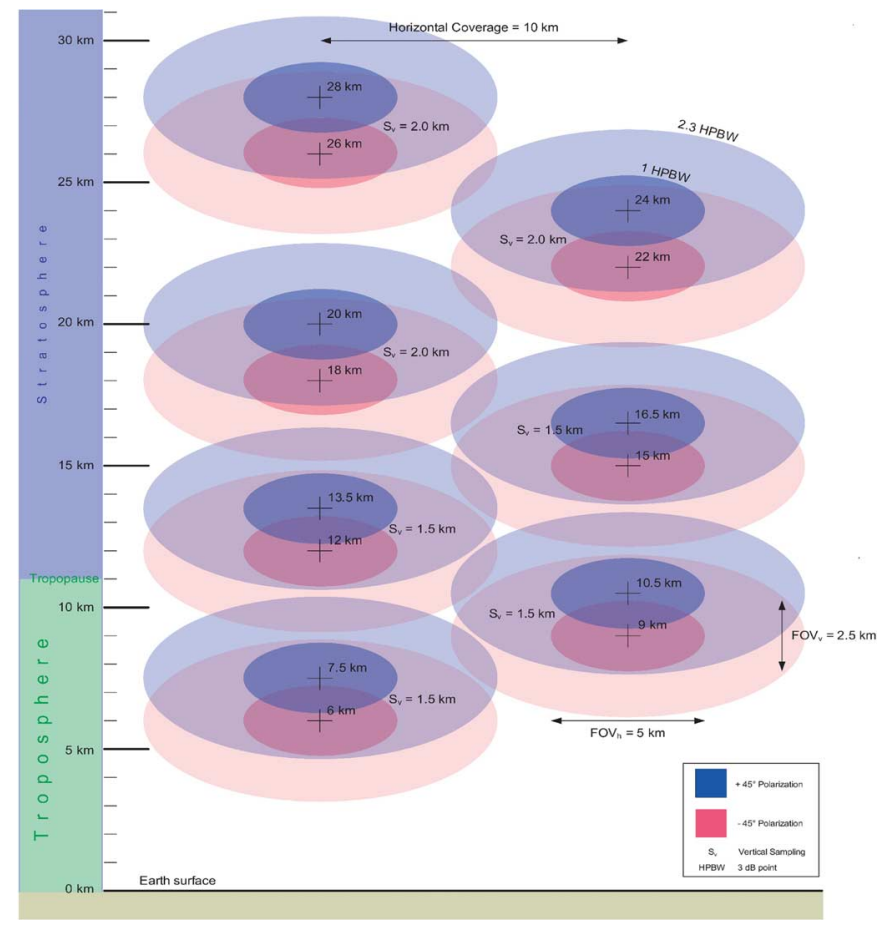

Fig. 1. Limb view geometry of the STEAMR antenna. The 14 observing beams are divided into two groups in terms of polarization, illustrated here as $+45^{\circ}$ polarisation in blue and $-45^{\circ}$ polarization in red.

date for the Earth Core Explorer mission [11]. The aim of the PREMIER mission (Process Exploration through Measurement of Infrared and millimeter-wave Emitted Radiation) will be to advance current understanding of the processes that link trace gases, radiation and chemistry in the upper troposphere and lower stratosphere.

The proposed baseline for the STEAMR instrument is a passive multi-beam limb sounder to observe in two $12 \mathrm{GHz}$ wide frequency bands between $323-335 \mathrm{GHz}$ and $343-355 \mathrm{GHz}$. The antenna will utilize a staring concept of 14 beams configured in a push broom geometry, covering a nominal altitude range of 6-28 km at mid-latitudes with an azimuthal spread of $10 \mathrm{~km}$ and are separated into two groups of seven beams, polarized at $\pm 45^{\circ}$.

The optical train of the STEAMR antenna, illustrated in Fig. 2, is treated in three distinct sections. The primary and secondary reflectors, referred to here on as $\mathrm{M} 1$ and $\mathrm{M} 2$, respectively, form an off-axis Ritchey-Chrétien telescope design, with both reflectors being paired hyperboloids of revolution. This particular telescope configuration is chosen for its larger field of view over the classic Cassegrain or Gregorian designs, as well as the inherent removal of third-order coma and spherical aberrations [12], thereby providing the best possible imaging requirements for the widely spread multiple observations beams. The primary aperture rim is elliptical, thereby defining the elliptical amplitude beam profiles of the limb views seen in Fig. 1. This thus sets the beams incident to the antenna as being astigmatic.

The subsequent four relay reflectors, referred to sequentially as M3, M4, M5, and M6, comprise a beam-forming network composed of an anastigmatic imaging network (M3, M4, M5),

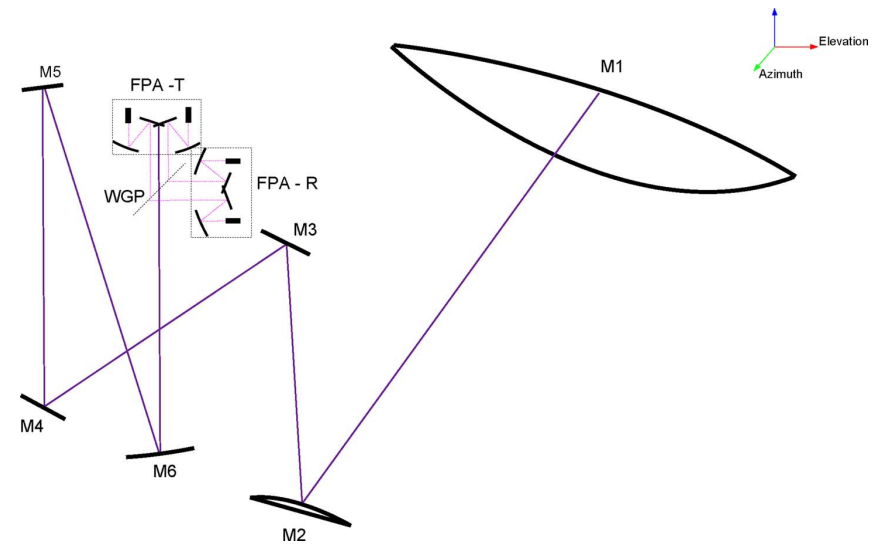

Fig. 2. Simplified ray-trace model of the STEAMR antenna optics, listing the component reflectors including the focal plane arrays (FPA), where the subscripts $\mathrm{T}$ and $\mathrm{R}$ refer to transmission and reflection through the wire grid polarizer (WGP).

plus one subsequent reflector (M6) required to provide the necessary phase shift between the antenna farfield and the output focal plane. This is a key requirement for a multi-beam antenna, wherein the beams must be intercepted at the location of maximum mutual separation. In this instance this requires that the output focal plane be located at a phase shift of $n \pi$ from the antenna far field. Accounting for the $\pi / 2$ phase shift between the primary aperture and the far field, this implies that the phase shift between the primary aperture plane and output focal plane be $n-\pi / 2$. This beam forming network thus provides circularly symmetric point spread functions across an unaberrated image of the sky at the output focal plane of the complete optical system. At this output focal plane, the beams then can be efficiently coupled to an array of feed horns through a focal plane array (FPA). Furthermore, the relay reflectors provide the required spatial accommodation for the internal calibration optics, which consists of an intercepting chopper reflector and associated optics to couple the receivers to the various loads and cold sky view, the details of which are not discussed further in this paper.

The final distinct section of the antenna optics is the FPA. This array intercepts the individual beams at the antenna output focal plane and re-images them to their corresponding feed horns. The optics for each beam within the FPA are equivalent, consisting of two paired off-axis conic reflectors, referred to from here on as M7 and M8. The imaging of this FPA is such that it provides the required additional $\pi / 2$ phase shift between the output focal plane and the feed horn apertures, thereby ensuring frequency independent coupling between the feed horn apertures and the primary antenna aperture plane.

\section{A. Anastigmatic Imaging Network}

The anastigmatic imaging network for the STEAMR beam forming network produces stigmatic Gaussian beams at the output plane of M5. These beams are then equivalently treated as circularly symmetric Gaussian beams as they evolve onwards in the optical train to be coupled to the feed horns in the FPA. Equivalently viewing the horns as radiators, thereby invoking reciprocity, the beam forming network coupled to the telescope 


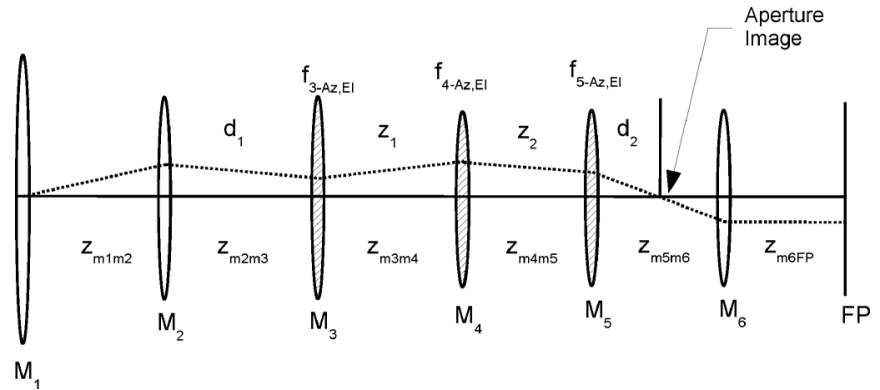

Fig. 3. Thin lens representation of the STEAMR antenna optics from the primary aperture (M1) through to the output focal plane (FP). The anastigmatic network, i.e., M3, M4 and M5 are shown as hatched filled lenses. Note the inclusion of the M6 reflector in order to produce the desired output focal plane FP.

should produce a set of elliptical beams on the sky with the desired aperture illumination. This requires that the radiated horn beams should therefore form flat phase fronts (i.e., beam waists) at the telescope aperture with beam widths consistent with the required edge tapers at that plane. The complete relay optics (M3-M6) are thus required to transform waists at the output focal plane to waists at the telescope aperture for the two orthogonal directions as well as matching the phase shifts for the two directions to ensure correct in-focus imaging at the output focal plane. Only in this way can correct imaging of the sky onto circularly symmetric beam patterns at the output focal plane be achieved.

We apply the technique described in Section II in order to determine the orthogonal focal lengths of the anastigmatic imaging network reflectors. For simplicity, the optical network is developed for the hypothetical on-axis chief beam rather than for a complete beam set, with the aim of locating reflectors at image planes of the primary aperture i.e., at positions of $n \pi$ phase shift from M1 so as to ensure that the beams are clustered about the central optical axis, thereby ensuring minimal off-center reflection distortions and reduce required reflector area. To fulfill the requirement for a $n-\pi / 2$ phase shift between the primary aperture plane and the output focal plane, we must ensure that the anastigmatic network produce an image of the primary aperture plane at an output distance $d_{2}$ from M5. This is an image of the telescope aperture i.e., a Fourier plane of the output image focal plane of the telescope. The final M6 reflector is thus located to produce the appropriate Fourier pattern of the beams at the output focal plane. The beam amplitude radius at the aperture image plane is $w_{F}$, where the beams are non-astigmatic and is given by $w_{F} w_{\text {out }}=\lambda f_{M 6} / \pi$, where $w_{\text {out }}$ is the beam amplitude radius at the output focal plane. With this framework in place it is possible to determine the independent $A B C D$ matrices $M_{\mathrm{Az}}$ and $M_{\mathrm{El}}$ that produces the desired matched output beam parameters. The complete antenna optics scheme, minus the FPA, is illustrated using a thin lens representation in Fig. 3.

The resultant focal length data and Gaussian beam parameter evolution for the resultant optics scheme is summarized in Table I. In Fig. 4 the evolution of the skew Gaussian beam width and phase shift throughout the complete STEAMR antenna optics is illustrated. As can be seen, the combination of reflectors M3 and M4 produce the required matching of amplitude and phase shift at the plane of the M5 reflector, beyond which the
TABLE I

Gaussian BeAm Parameters and Focal Lengths of STEAMR COMPONENT REFLECTORS AT THE CENTRAL OPERATING FREQUENCY OF $340 \mathrm{GHz}$

\begin{tabular}{|c|c|c|c|c|c|c|}
\hline Mirror & $\begin{array}{c}w_{A z} \\
{[\mathrm{~mm}]}\end{array}$ & $\begin{array}{c}w_{E l} \\
{[\mathrm{~mm}]}\end{array}$ & $\begin{array}{c}f_{A z} \\
{[\mathrm{~mm}]}\end{array}$ & $\begin{array}{c}f_{E l} \\
{[\mathrm{~mm}]}\end{array}$ & $\begin{array}{c}\Delta \phi_{A z} \\
{[\mathrm{rad}]}\end{array}$ & $\begin{array}{c}\Delta \phi_{A z} \\
\text { [rad] }\end{array}$ \\
\hline M1 & 235.75 & 471.5 & \multicolumn{2}{|c|}{1105.86} & \multicolumn{2}{|c|}{0.0} \\
\hline M2 & 29.09 & 58.1302 & \multicolumn{2}{|c|}{-151.46} & 0.04 & 0.01 \\
\hline M3 & 19.35 & 37.2 & -2150.1 & $-1.72 \times 10^{4}$ & 0.289 & 0.074 \\
\hline M4 & 12.83 & 23.56 & 542.81 & 681.0 & 0.98 & 0.256 \\
\hline M5 & \multicolumn{2}{|c|}{14.08} & 490.61 & 254.76 & \multicolumn{2}{|c|}{2.84} \\
\hline Image Plane & \multicolumn{2}{|c|}{14.74} & 490.61 & 254.76 & \multicolumn{2}{|c|}{$3.14=\pi$} \\
\hline M6 & \multicolumn{2}{|c|}{20.48} & \multicolumn{2}{|c|}{526.37} & \multicolumn{2}{|c|}{-2.64} \\
\hline Focal Plane & \multicolumn{2}{|c|}{10.02} & \multicolumn{2}{|c|}{$\mathrm{n} / \mathrm{a}$} & \multicolumn{2}{|c|}{-1.52} \\
\hline
\end{tabular}

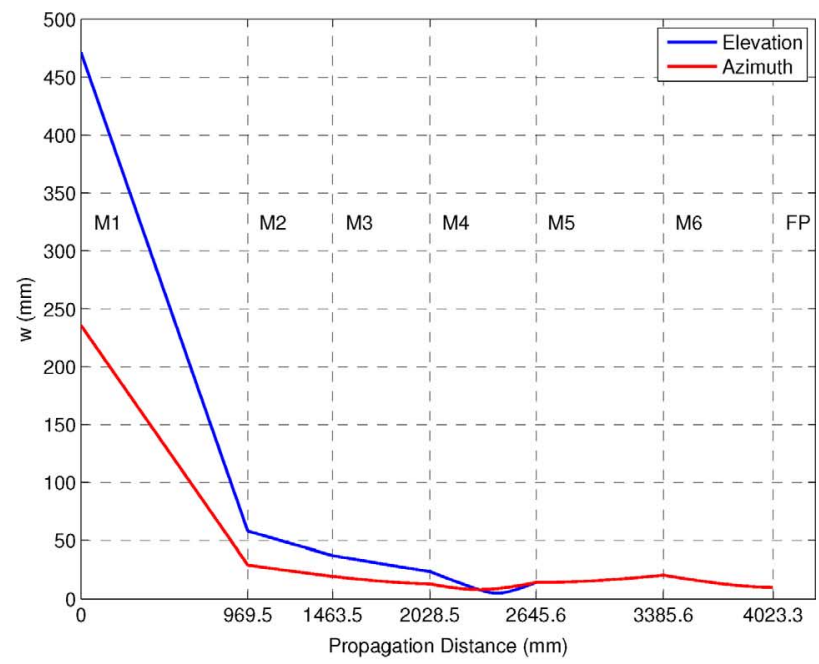

(a)

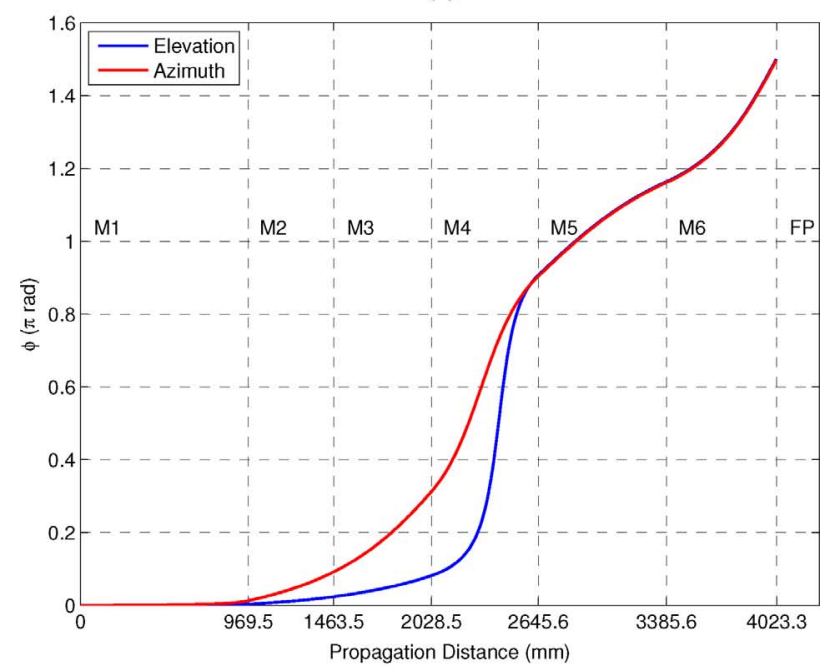

(b)

Fig. 4. Evolution of Gaussian beam amplitude radius (a) and Guoy phase shift (b) for the STEAMR antenna optics at the central frequency of $340 \mathrm{GHz}-\mathrm{cf}$. Table I. The phase shift is given in absolute value, with the phase jump from $+\pi$ to $-\pi$ at the image plane occurring at $\pi$ radians after M5. (a) Beam width. (b) Phase shift.

beams evolve equivalently for the two orthogonal directions, thereby forming a circularly symmetric, stigmatic beam.

It should be emphasized here that while a particular anastigmatic network solution may be determined and verified with Gaussian beam analysis, the practical implementation of this imaging network using off-axis astigmatic reflectors is not as straightforward. It should be ensured that the conic parameters 
of the reflectors in the two orthogonal planes be relatively similar, and be of the same type, so that that the surface transition between the two orthogonal off-axis surfaces is as smooth as possible. Furthermore, the conic parameters of the orthogonal surfaces should be such that their respective conic vertices are spaced sufficiently far apart. This requirement can be achieved by varying the inter-mirror distances until a suitable solution is calculated. In the case of the M5 reflector for the STEAMR anastigmatic network presented here, the vertices of the two orthogonal paraboloids are spaced close enough such that a "cusplike" discontinuity structure is formed between them. While this forms a technically undesirable reflecting surface, the resultant beams formed by the complete antenna optics meet the antenna performance requirements.

\section{Astigmatic ReLay RefLectors}

To successfully translate the theoretical anastigmatic network detailed in Section III-A into a physical application requires utilising reflectors with the desired focal lengths in the azimuth and elevation axes. Since the entire STEAMR optics scheme utilizes off-axis reflectors, standard on-axis biconic, or toroidal reflectors [12] are insufficient here. We utilize the techniques developed in [13], wherein an efficient technique to describe toroidal reflectors with off-axis geometries, referred to as astigmatic off-axis reflectors, is given. We summarize briefly the technique employed as it pertains to the STEAMR reflectors, and the reader is directed to the source material for the complete general description.

The process to describe a standard rotationally symmetric off-axis conic section for reflection of a Gaussian beam, be it an ellipsoid, hyperboloid or paraboloid, requires matching the incident phase radius of curvature, $R_{\mathrm{in}}$ to the desired output phase radius of curvature, $R_{\text {out }}$, through a prescribed half reflection angle $\theta_{i}$. From these three values the geometrical construction of the parent conic may then be determined using standard relations [12]. For the construction of astigmatic off-axis reflectors, [13] considers the construction of the conic section in two orthogonal directions, dependent upon the geometry of the system in question. Whereas a standard off-axis reflector is considered to transfer the so-called phase center, i.e., beam waist, of the incident beam to the phase center for the desired output beam, astigmatic reflectors can control either three or four phase centers simultaneously. Indeed, it can be further said that all offaxis astigmatic reflectors are four-phase, being that the threephase reflector is a special case where either the two input or two output phase centers are collocated. These reflectors are thus divided into two types, according to their function. The fourphase off-axis astigmatic transforms an elliptical beam, with two phase centers, to another elliptical beam, also with two phase centers. The three-phase off-axis astigmatic transforms an ellipsoidal beam, with two phase centers, to a circularly symmetric beam, with one phase center, and vice versa. In this case the reflector surface must be located where the beam itself is circular, as the mirror only modifies phase and not the amplitude distribution. These reflectors thus provide orthogonal focussing properties $\left(f_{x}, f_{y}\right)$. Both types of astigmatic off-axis reflector will be required for the anastigmatic section of the STEAMR optics train.

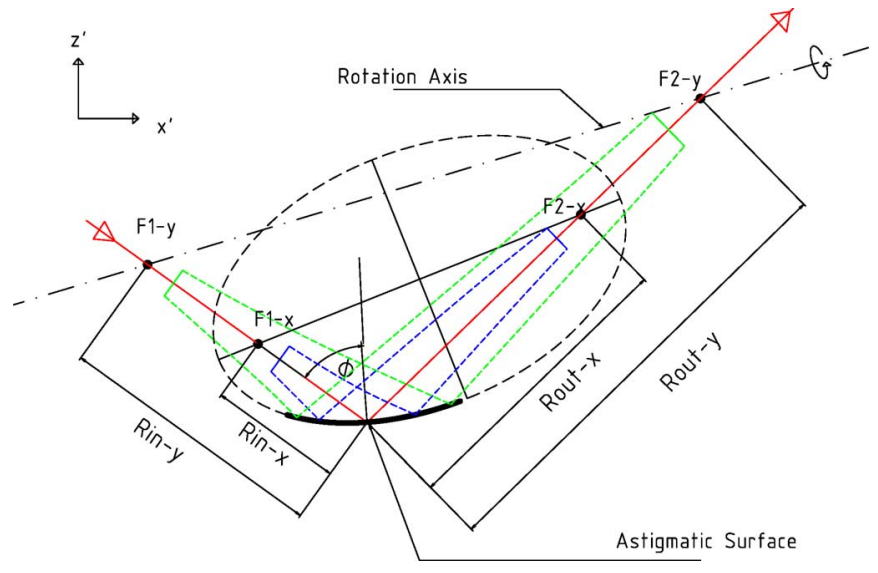

Fig. 5. Graphic representation of generation of four-phase astigmatic reflector with both orthogonal conic definitions being off-axis ellipsoids. The $\left(x^{\prime}, z^{\prime}\right)$ plane defines the global plane of the conic defined by $\left(R_{\mathrm{in}-x}, R_{\text {out }-x}\right)$. The evolutions of the orthogonal Gaussian beams in the two directions are given in blue (x) and green (y).

TABLE II

INPUT AND OUtPUt PHASE RADII OF CURVATURE AND HALF REFLECTION ANGLE FOR Astigmatic OfF-AXIS REFLECTORS M3, M4 AND M5 Calculated at Central Frequency (340 GHz)

\begin{tabular}{|c|c|c|c|c|}
\hline $\mathrm{M} 3$ & $R_{\text {in }}[\mathrm{mm}]$ & $R_{\text {out }}[\mathrm{mm}]$ & $\theta\left[{ }^{\circ}\right]$ & Conic \\
\hline $\mathrm{Az}$ & -1081.23 & -1153.69 & 29.76 & Convex Hyperboloid \\
$\mathrm{El}$ & -883.095 & -1498.62 & 29.76 & Convex Hyperboloid \\
\hline $\mathrm{M} 4$ & $R_{\text {in }}[\mathrm{mm}]$ & $R_{\text {out }}[\mathrm{mm}]$ & $\theta\left[{ }^{\circ}\right]$ & Conic \\
\hline $\mathrm{Az}$ & -3523.99 & -470.359 & 28.0 & Concave Hyperboloid \\
$\mathrm{El}$ & -1022.36 & -408.738 & 28.0 & Concave Hyperboloid \\
\hline $\mathrm{M} 5$ & $R_{\text {in }}[\mathrm{mm}]$ & $R_{\text {out }}[\mathrm{mm}]$ & $\theta\left[{ }^{\circ}\right]$ & Conic \\
\hline $\mathrm{Az}$ & 490.048 & $\infty$ & 17.75 & Concave Paraboloid \\
$\mathrm{El}$ & 254.151 & $\infty$ & 17.75 & Concave Paraboloid \\
\hline
\end{tabular}

As before, a skew Gaussian beam is treated with orthogonal beam parameters $\left(w_{x}, R_{x}, \theta_{x}\right)$ and $\left(w_{y}, R_{y}, \theta_{y}\right)$, which accordingly do not originate from the same phase center, thereby ensuring that $R_{\mathrm{in}-x} \neq R_{\mathrm{in}-y}$. In a four-phase astigmatic reflector the output beam will also be elliptical $\left(R_{\text {out }-x} \neq R_{\text {out }-y}\right)$, whereas for a three-phase astigmatic the output beam will be circularly symmetric $\left(R_{\text {out }-x}=R_{\text {out }-y}\right)$.

The construction of a four-phase astigmatic reflector is illustrated in Fig. 5. In this example the curve locus for the conic section in the global $\left(x^{\prime}, z^{\prime}\right)$ coordinate frame is defined in the standard way between the two focal points $(F 1-x, F 2-x)$. The major axis for the orthogonal conic section, defined between the focal points $(F 1-y, F 2-y)$ is then drawn. The final surface is then achieved through rotation of the x-plane curve locus about the $v$-plane curve major axis. This technique results in a smooth transition from the $x$ conic locus to the $y$ conic locus.

The conic parameter data for the STEAMR astigmatic reflectors in Table II is derived from the Gaussian beam parameters presented in Table I. Accordingly, we see that reflectors M3 and M4 are four-phase astigmatic reflectors, the combination of which transforms the incident elliptical beam from the M1-M2 telescope to a beam with a circularly symmetric amplitude distribution and matched Guoy phase shifts at the plane of the M5 reflector. The M5 reflector, as can be seen from the equivalent output phase radii of curvature for the azimuth and elevation directions above in Table II, is a three-phase off-axis 
astigmatic. It is the addition of this last reflector that ensures the matching of the phase radii of curvature in the orthogonal planes, thereby completing the anastigmatic optical network. Note here that the requirement for similar conic parameters for the orthogonal planes is satisfied.

\section{Focal Plane Array (FPA)}

The final component of the STEAMR optical train is the focal plane array (FPA). The layout of the FPA, as illustrated in Fig. 2, shows the two imaging arrays FPA-T and FPA-R, where $T$ and $\mathrm{R}$ stand for transmission and reflection respectively, and the adjoined wire grid polariser (WGP), with wires oriented at a projected $45^{\circ}$ with respect to the plane orthogonal to the optical axis, separates the incident 14 beams into the two groups of seven. The optics of each pixel in both arrays is equivalent, with the specific pointing being determined from geometrical ray tracing calculations through the complete optics train.

The construction of the FPA is based primarily upon the heritage of two similar array systems [14] and [15] wherein the individual pixel reflectors are formed as a monolithically machined facet reflector array. This FPA design provides significant performance improvement over an array of direct feed horns at the focal plane. Firstly, in locating the focal plane at a phase shift of $n-\pi / 2$ from M1 the beams are at their most frequency dependent. Thus a direct-fed feed horn array would produce varying edge tapers on the M1 reflector across the frequency band, leading to frequency dependent beam field of view on the sky. Secondly, the mutual separation between the beams at the focal plane is not sufficiently large enough to accommodate the individual feed horns. The feed horn aperture radii could potentially be reduced significantly to account for this, however this would result in an undesirably low feed horn gain. Furthermore, this would also alter the edge taper on the primary aperture and subsequently the beam field of view. For these reasons it is considered more efficient to control the incoming beams optically in order to couple them to the corresponding feeds.

The primary challenge in the design of the FPA is achieving maximum spillover efficiency for each beam. The vertical sampling of the beams in the far field directly determines the maximum mutual separation, and hence the maximum achievable spillover efficiency. The observing requirements for STEAMR, as is typical in millimeter-wave radiometer antennas, places a higher priority on beam efficiency over antenna pattern resolution [16], [17], and as such the edge taper of the primary aperture is $25 \mathrm{~dB}$ in terms of 1-D fundamental Gaussian beam illumination. This degree of aperture illumination combined with the vertical spatial sampling of the observing beams in the farfield plane translates to a beam proximity in terms of beam amplitude radius of approximately $18 \mathrm{~dB}$. In terms of power conservation, this is a significant limitation over the typical standard for near field quasi-optical imaging, where a minimum beam truncation radius of the order of $35 \mathrm{~dB}$ is expected, thus ensuring power throughput of $99.97 \%$ for the fundamental Gaussian beam [5], compared to the $98.42 \%$ for an $18 \mathrm{~dB}$ radius. Furthermore, these values are calculated for the fundamental Gaussian, and do not consider the additional power lost to sidelobes. The rims of the $\mathrm{M} 7$ facet reflectors have thus been designed to maximize

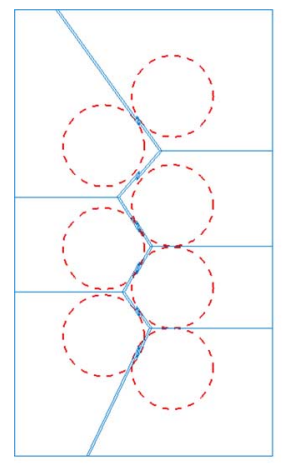

(a)

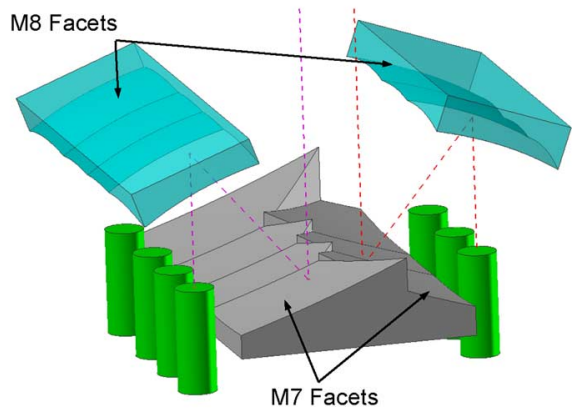

(b)
Fig. 6. Focal plane array M7 facet reflector geometry (a), showing individual beam footprints for the $18 \mathrm{~dB}$ radius at the central frequency $(340 \mathrm{GHz})$. The rims for the individual facets are designed in order to accommodate maximum individual beam throughput. A 3D CAD model of the complete array optics is shown in (b), showing the M7 and M8 facets and the individual feed horns.

power throughput for the individual beams. Owing to the relatively long focal length for the M7 conic, this leads to a complex 'saw-tooth' facet geometry.

In order to minimize potential truncation and diffraction losses at the M7 facet reflector it is necessary to employ a feed horn with a highly centralized aperture field distribution and low side lobes. The design for the STEAMR feed horns is based upon that given in [18], referred to here as "ultra-Gaussian." The ultra-Gaussian horn employs a profiled corrugated feed design to excite both the $H E_{11}$ and $H E_{12}$ modes, followed by an additional cylindrical waveguide section of appropriate length to match the phases of these two modes. The chosen amplitudes of these two hybrid modes produces an aperture distribution with significantly low sidelobe levels at $\simeq 40 \mathrm{~dB}$ thereby maximising the power coupling coefficient to the representative fundamental Gaussian beam at $99.8 \%$ [18]. A full treatment of a breadboard model of this FPA is given in [19] and [20].

\section{Electromagnetic Simulations}

The complete STEAMR antenna optics train has been modeled using the reflector antenna design tool GRASP developed by TICRA. This package utilizes the numerical approximation techniques of physical optics (PO), physical theory of diffraction (PTD), and method of moments (MoM) to provide highly accurate simulations of long-wavelength reflector antenna systems. From these analysis data we can draw extremely reliable conclusions on the success of the antenna optics model. As per the limb-viewing geometry requirements of the antenna, in the following analyses of these simulated data importance is placed on the beam pattern parameters for the elevation direction over azimuth.

In Fig. 7, simulated farfield contour plots for the complete beam set are presented. Overall the beams exhibit the desired elliptical patterns, with the outermost beams in the upper and lower ranges displaying a minor degree of rotation in the positive and negative elevation directions respectively, with the centrally locate beams displaying the highest conformity with desired patterns in Fig. 1. 


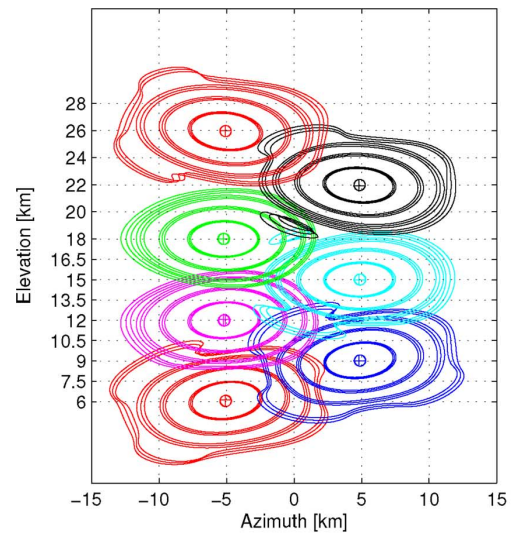

(a)

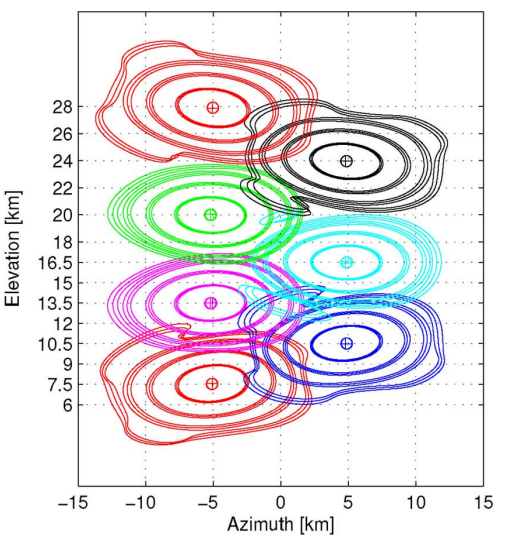

(b)

Fig. 7. Farfield contour plots for (a) $\mathrm{T}$ beams and (b) $\mathrm{R}$ beams calculated from PO simulations. Contour levels spaced at $-3,-10,-20$, and $-30 \mathrm{~dB}$ for frequencies 323, 340, and $357 \mathrm{GHz}$.

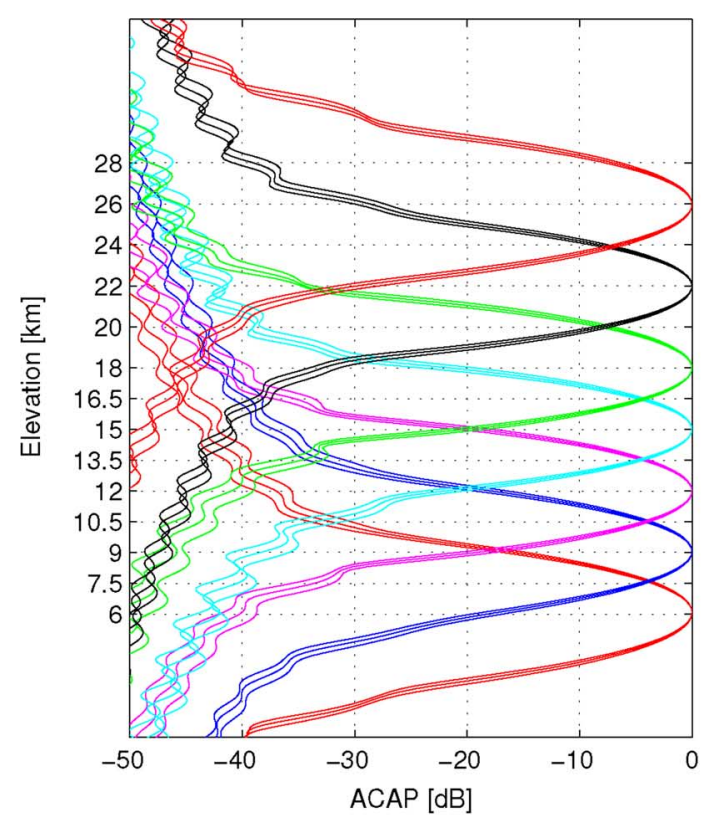

(a)

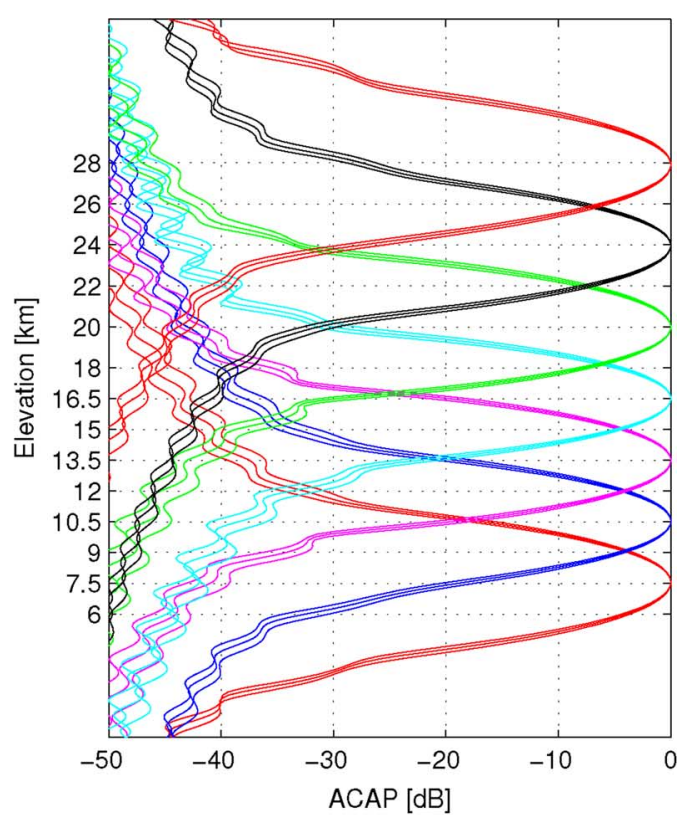

(b)

Fig. 8. ACAPs for the (a) T beams and (b) R beams for with three contours per beam for frequencies 323, 340, and $357 \mathrm{GHz}$.

Specific antenna performance requirements necessitate that the azimuthally collapsed antenna patterns (ACAPs) first sidelobe amplitudes be $\leq-30 \mathrm{~dB}$. The ACAPs are generated through numerically integrating the individual beams across the azimuth direction. In Fig. 8, the ACAPs for the complete beam set are shown, and as is clearly evident each beam fulfills this requirement. Additionally, the maximum first sidelobe amplitude level for the azimuth and elevation planes through the beam centers is $\sim-43 \mathrm{~dB}$.

The maximum threshold for the field of view full-width at half-maximum (FWHM) is specified at $\leq 3 \mathrm{~km}$, with a goal of $\leq 2 \mathrm{~km}$. From the FWHM data illustrated in Fig. 9(a), it is shown that all beams meet this requirement. The effect of amplitude pattern distortion is further evident here as increased FWHM values for the beams in the upper and lower elevation ranges. The FWHM requirements for the azimuthal direction are $\leq 10 \mathrm{~km}$, which are well met. Accurate pointing knowl- edge of the beams is required, specifically in the elevation direction, in order to correctly determine the individual beam viewing scene. Residual pointing values, calculated from comparisons with nominal pointing values, are shown in Fig. 9(b).

The main beam efficiency, $\eta_{\mathrm{mb}}$, of the antenna, being the integrated power in the main beam relative to the total power received by the antenna, is stipulated to a minimum of $85 \%$ and a maximum of $95 \%$. In Fig. 10 the residual $\eta_{\mathrm{mb}}$ values (i.e., $1-\eta_{\mathrm{mb}}$ ) are plotted against incremented values of $\Omega_{\mathrm{mb}}$ for a single set of beams at the central frequency. In lieu of using the complete $4 \pi$ sphere for normalisation, a solid angle of sufficient size for each beam was selected. The value for FWHM has been determined for each individual beam from PO calculations and the values are noted in the corresponding legends.

These data are representative of the $\eta_{\mathrm{mb}}$ for the complete beam set of STEAMR over the frequency band and show that the beams sufficiently fulfill the $\eta_{\text {mbe }}$ requirement. The relative 


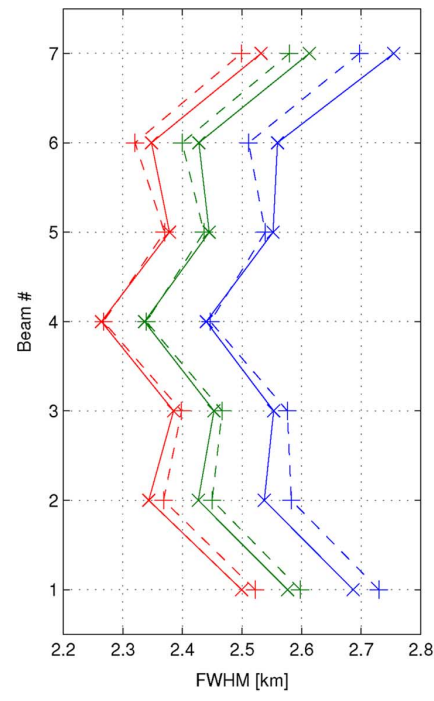

(a)

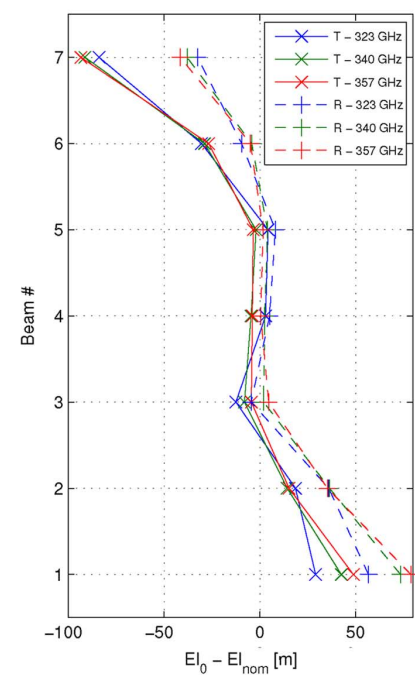

(b)
Fig. 9. FWHM data for (a) each beam and (b) elevation pointing offset normalized to the nominal pointing.

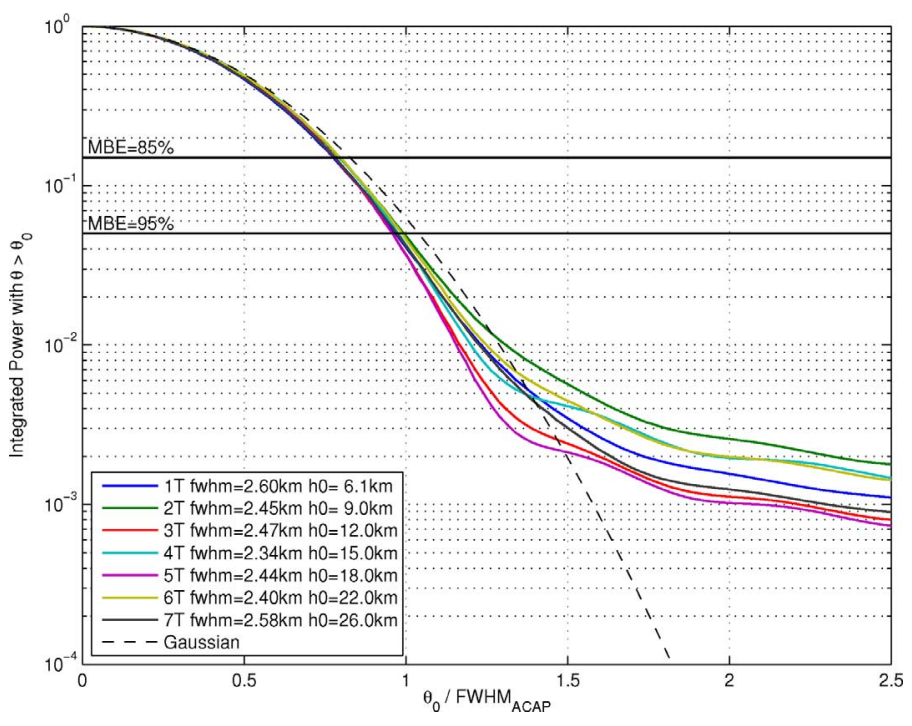

Fig. 10. Numerically integrated values for $1-\eta_{\mathrm{mb}}$ as a function of increasing $\Omega_{\mathrm{mb}}$ for a single beam set at the central frequency of $340 \mathrm{GHz}$. The corresponding data for a representative fundamental Gaussian beam is also shown.

power throughput for the individual beams, determined numerically from GRASP simulations, is displayed in Fig. 11 for a single beam set at the lower, central and upper frequencies. As expected, the $\mathrm{M} 7$ facet reflector, being the intercepting reflector at the focal plane, shows the highest degree of truncation. Despite this, the minimum relative power conservation occurring at the lowest frequency remains high at $>98.0 \%$. The truncation of the M7 facet dominates the subsequent power throughput for the individual beams, with the variance in conserved power between the beams being due to the unequal truncation of the beams at the M7 facet, as shown in Fig. 6(a). Similar simulations of conserved power throughput where the M7 facet reflector is oversized reveals this, and the edge taper values for all beams are equivalent, thus revealing the frequency independent imaging of the complete antenna optics.

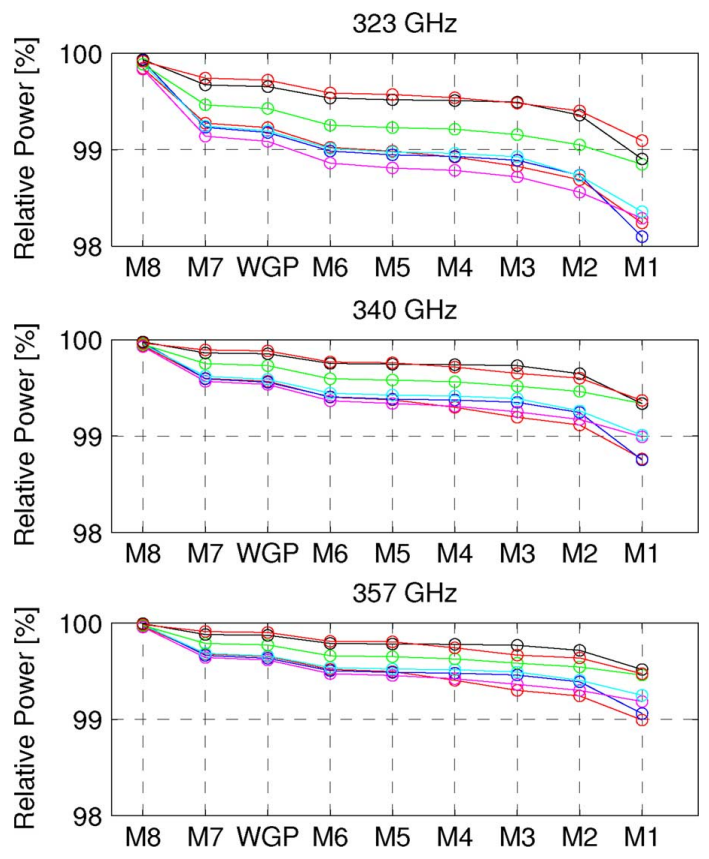

Fig. 11. Relative power conservation throughout the complete antenna optics train for a single beam set at 323,340 , and $357 \mathrm{GHz}$, respectively. The dominant truncation from the M7 facet reflector is evident here.

\section{CONCLUSION}

A novel technique for the removal of simple astigmatism in Gaussian beams through optical means has been presented. A theoretical framework for an anastigmatic imaging network composed of off-axis reflecting optics using $A B C D$ matrices in conjunction with Gaussian beam imaging has been shown to successfully remove the inherent astigmatism of this optics scheme, thereby ensuring the desired amplitude and phase parameters necessary for optimum imaging between the antenna and the focal plane. This technique has been successfully implemented as part of a complete optical model for the STEAMR antenna with the GRASP software package. The optical performance of this model, as illustrated the resultant farfield beam pattern data presented in Figs. 7 and 8, shows excellent conformance with prescribed requirements. This thus serves as a strong validation of the theoretical technique presented in Section II.

The results of this optical design and analysis work reveal several important conclusions. Firstly, an anastigmatic imaging network can be successfully incorporated into an off-axis optics scheme. Furthermore, the design process considered only a single chief ray for solution of this imaging requirement, which was then successfully extended for a multi-beam framework, thereby making the design process for this multi-beam system more efficient. This imaging network principle could furthermore be extended to similar imaging systems that utilize lenses, the results of which would preclude the inherent distortions from off-axis reflectors. The technique can also be readily applied to single beam antennas, which would not require a focal plane array system and thus simplify the imaging requirements. Secondly, the high mutual proximity of the observing beams has 
been shown to not be detrimental to power conservation requirements of the antenna, despite the unavoidable frequency dependent illumination of the FPA facet reflectors, the net effect in terms of power throughput is negligible.

\section{ACKNOWLEDGMENT}

The authors would like to thank E. Jorgensen from Ticra for providing support in importing of CAD surfaces for the STEAMR FPA model.

\section{REFERENCES}

[1] Y. Irimajiri et al., "BSMILES: A balloon-borne superconducting submillimeter-wave limb-emission sounder for stratospheric measurements," IEEE Geosci. Remote Lett., vol. 3, no. 1, pp. 88-92, Jan. 2006.

[2] R. E. Cofield and P. C. Stek, "Design and field-of-view calibration of $114660-\mathrm{GHz}$ optics of the earth observing system microwave limb sounder," IEEE Geosci. Remote Lett., vol. 44, no. 5, pp. 1166-1181, May 2006.

[3] J. Alda, Encyclopedia of Optical Engineering: Laser and Gaussian Beam Propagation and Transformation. Boca Raton, FL: CRC Press, 2003, ch. 13.

[4] A. Bruschi, S. Cirant, G. Granucci, A. Simonetto, and G. Solari, "Conditioning optics for astigmatic Gaussian beams at $140 \mathrm{GHz}, 0.5 \mathrm{MW}$," Int. J. Infrared Millim. Waves, vol. 15, pp. 1413-1420, 1994.

[5] P. F. Goldsmith, Quasioptical Systems. Piscataway, NJ: IEEE Press, 1998.

[6] R. Wylde, "Millimetre-wave Gaussian beam-mode optics and corrugated feed horns," in Proc. IEE Microwaves, Optics and Antennas, Aug. 1984, vol. 131, no. 4, pp. 258-262.

[7] J. A. Arnaud and H. Kogelnik, "Gaussian light beams with general astigmatism," Appl. Opt., vol. 8, no. 8, pp. 1687-1693, Aug. 1969.

[8] S. Yang, M. Bai, and J. Miao, "Deastigmatism and circularization of an elliptical Gaussian beam by off-axis ellipsoid reflector based off-focus configuration," Progr. Electromagn. Res. B, vol. 10, no. 3, pp. 91-103, 2008.

[9] P. Goldsmith, "Gaussian beam transformation with cylindrical lenses," IEEE Trans. Antennas Propag., vol. 34, no. 4, pp. 603-607, Apr. 1986.

[10] J. Serna and G. Nemeş, "Decoupling of coherent Gaussian beams with general astigmatism," Opt. Lett., vol. 18, no. 21, pp. 1774-1776, Nov. 1993.

[11] European Space Agency (ESA), "PREMIER: Process exploration through measurements of infrared and millimetre-wave emitted radiation," Nov. 2008 [Online]. Available: http://esamultimedia.esa.int/docs/SP1313-5-PREMIER.pdf

[12] R. N. Wilson, Reflecting Telescope Optics I. Reading, MA: Springer, 1972.

[13] A. Wagner-Gentner, U. Graf, M. Philipp, and D. Rabanus, "A simple method to design astigmatic off-axis mirrors," Infrared Phys. Technol., vol. 50 , no. 1 , pp. $42-46$, Nov. 2007.

[14] T. Luethi, D. Rabanus, U. U. Graf, C. Granet, and A. Murk, "CHARM - A compact heterodyne array receiver module for KOSMA with scalable fully rectective focal plane array optics," in Proc. 79th Annu. Scientific Meeting of Astronom. Gesellschaft, Cologne, Germany, 2005, vol. 326, no. 7.

[15] U. U. Graf et al., "SMART: The KOSMA sub-millimeter array receiver for two frequencies," in Proc. SPIE, Millim. Submillim. Detectors for Astronomy, 2003, vol. 4855, pp. 322-329.

[16] Y. Lo and S. Lee, Antenna Handbook III: Antenna Applications, 1st ed. New York: Springer, 1993, vol. 2.

[17] N. Skou and D. L. Vine, Microwave Radiometer Systems: Design and Analysis, 2nd ed. Reading, MA: Artech House, 2006, vol. 2.

[18] P. A. S. Cruickshank, D. R. Bolton, D. A. Robertson, R. J. Wylde, and G. M. Smith, "Reducing standing waves in quasi-optical systems by optimal feedhorn design," in Joint 32nd Int. Conf. on Infrared Millim. Waves, 2007, and 2007 15th Int. Conf. on THz Electron. , Sept. 2007, pp. 941-942.

[19] M. Renker, M. Whale, A. Murk, J. A. Murphy, and R. Wylde, “Analysis and simulation of edge effects of focal plane array facet reflectors with high filling factor," in 33rd ESA Antenna Workshop on Challenges for Space Antenna Systems, Oct. 2011, vol. 4855.

[20] M. Renker, M. Whale, and A. Murk, "Antenna simulations and measurements of focal plane array facet reflectors," IEEE Trans. Antennas Propag., submitted for publication.

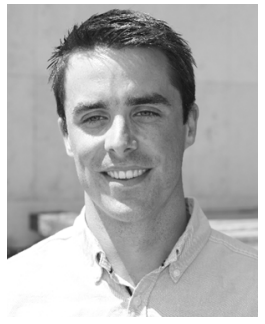

Mark Whale (M'11) received the B.Sc. degree (Hons.) in physics with astrophysics and the Ph.D. degree in submillimeter-wave optics from the $\mathrm{Na}$ tional University of Ireland, Maynooth, Co. Kildare, Ireland in 2005 and 2010, respectively.

During his Ph.D. research he collaborated extensively with the Space Research Organisation of the Netherlands (SRON), Groningen, the Netherlands, and the Group for Advanced Receiver Development (GARD), Chalmers Technical University, Gothenburg, Sweden, on design and optimization aspects of the front end optics of the Atacama Large Millimetre Array (ALMA) receiver bands 5 and 9 . He is currently a Post-Doctoral research assistant at the Microwave Physics Group at the Institute for Applied Physics, University of Bern, Bern, Switzerland. His primary research interests are in submillimeter-wave optical systems for astronomical and aeronomy antenna platforms. In 2010, he joined the University of Bern, Bern, Switzerland, where his chief responsibility has been the design, development and testing of a prototype focal plane array imaging system for the STEAMR instrument, as well as being involved in the design of the antenna's complete optical system.

Dr. Whale is a member of the Institute of Physics.

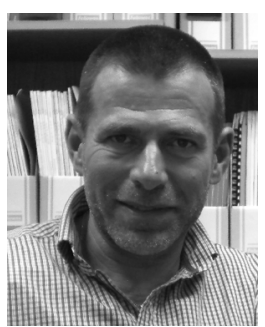

J. Anthony Murphy (S'04-M'11) received the B.Sc. and M.Sc. degrees in experimental physics in 1977 and 1979, respectively, from University College Cork, and also the M.S. degree in physics from the California Institute of Technology, Pasadena, in 1981, and the Ph.D. degree in physics from University of Cambridge, U.K., in 1986.

From 1985 to 1987, he worked as a Post-Doctoral Research Associate at the Cavendish Laboratory, University of Cambridge, U.K., on receiver development for the James Clerk Maxwell Telescope (Hawaii). In 1988, he became a member of the lecturing staff of the Experimental Physics Department at Maynooth. He is currently Professor and Head of the Department of Experimental Physics at the National University of Ireland, Maynooth, Ireland. His main research interests are in the area of far-infrared space optics and experimental cosmology, specifically, the cosmic microwave background. He was involved in the development of receiver systems for the European Space Agency Planck Surveyor Satellite and the Herschel Space Observatory.

Dr. Murphy is a member of the Institute of Physics, (CPhys, MInstP), and a Fellow of the Royal Astronomical Society.

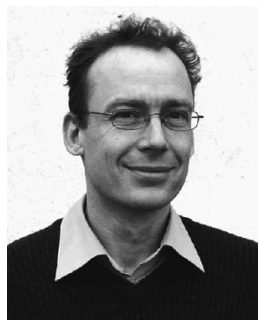

Axel Murk was born in Germany in 1968. He received the M.S. degree in physics from the Technical University of Munich, Munich, Germany, in 1995 and the Ph.D. degree in physics from the University of Bern, Bern, Switzerland, in 1999.

Since 1999, he has been involved in the development and characterization of submillimeter-wave instrumentation for different ground-based and spaceborne projects. In 2003, he was an Invited Scientist with the Japan Aerospace Exploration Agency (JAXA). Since 2006, he has been the Head of the Terahertz Optics Group, Institute of Applied Physics, University of Bern, Switzerland.

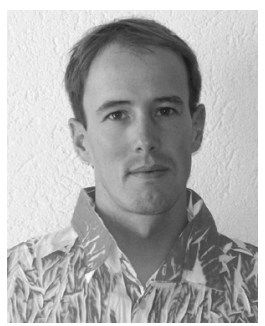

Matthias Renker received the B.Sc. degree in mechanical engineering at the University of Applied Sciences, Bern, Switzerland, in 1977, and also the B.Sc. and M.Sc. in physics with astrophysics studying in the Laser Department at IAP Bern in 2008 and 2009, respectively, and is currently working toward the Ph.D. degree from the Microwave Physics Group, Institute for Applied Physics (IAP), University of Bern, Bern, Switzerland. His Ph.D. research consists of analyses and measurements for the STEAMR instrument, ranging from mechanical design aspects and receiver breadboard testing.

He joined Creaholic SA as an engineer in 1998 where he was part of several industrial patents, as well as several successful industrial innovations, primarily in the automotive, medical, watchmaking and food packaging industries. There, he developed significant expertise in lightweight CFRP structures as well as nonlinear simulations pertaining to mechanical structures. 


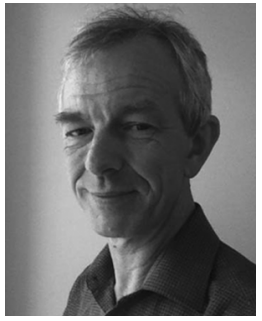

Urban O. Frisk received the Ph.D. degree in astronomy from the Astronomy Department of Stockholm University, in 1983.

He joined the ESA Space Science Department, one year as research fellow, and from 1984, as staff scientist. While at ESA, he acted as study scientist for the proposed space VLBI project QUASAT and project scientist for the FIRST/Herschel project. He developed an IR imaging spectrograph and a $500 \mathrm{GHz}$ heterodyne spectrometer. The work included participation in defining and selecting, and supporting industrial studies and technical development for future ESA scientific projects. These have been carried out supported by scientific advisory groups. Participation and organization of workshops and conferences related to the ESA science program and in conjunction, write-ups and presentations. From 1991, he was with the Swedish Space Corporation (SSC) as one of the original proposers of the combined aeronomy and astronomy mission, Odin. He was the SSC project scientist and also acted as the payload manager. Odin carries tunable 500-600 GHz cryogenic heterodyne receivers in addition to a fixed MMIC based at $119 \mathrm{GHz}$, was launched in 2001 and on its 12 th year is still fully operational. He participated in the Core Earth Explorer proposal to ESA in 2005 for PREMIER, aimed to understand atmospheric processes linking trace gases, radiation, chemistry and climate, with one of the instruments, STEAMR supplied by Sweden and is still working to develop this instrument, and in 2011 started to work for Omnisys Instruments. He is currently responsible for the scientific planning of Odin and is also active in developing instrumentation and future scientific missions.

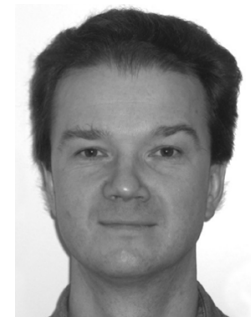

Olle Janson worked as a mechanical design engineer at Saab Aircraft from 1982. At the Military Aircraft division he spent the first 7 years in hydraulics and landing gear design on the JAS 39 Gripen multirole fighter aircraft. In 1989, after switching to Commercial Aircraft, he became principal designer of the hydraulics for the Saab 2000 airliner. After a 4-year period in the nuclear power business he returned to aerospace in 2000, joining the Swedish Space Corporation (SSC). Here, working in collaboration with instrument principal investigators, he has worked on mechanical design for microgravity experiment payloads such as ITEL, BIG, FOAMS and BIOMICS within the ESA sounding rocket program. Between 2005 and 2010, he developed the mechanical designs of the NanoSpace MEMS Micropropulsion system for the PRISMA satellite. He is also responsible for the companys mechanical CAD system. Since 2007 , he has been chiefly involved in the primary mechanical and optical design of the STEAMR instrument. 